\title{
Ecomorphological, behavioural and physiological patterns in otoliths
}

\author{
Q. Palazzo ${ }^{1,6}$, M. Stagioni ${ }^{2}$, S. Raaijmakers ${ }^{3}$, R.G. Belleman ${ }^{3}$, F. Prada ${ }^{4,6}$, S. Fermani ${ }^{1}$, J.U. Hammel ${ }^{5}$, J. Kaandorp ${ }^{3}$, \\ S. Goffredo ${ }^{4,6}$, G. Falini ${ }^{1,6}$ \\ ${ }^{1}$ Department of Chemistry “Giacomo Ciamician”, University of Bologna, Via Selmi 2, 40126 Bologna, Italy, \\ ${ }^{2}$ Laboratory of Fisheries and Marine Biology at Fano, Department of Biological, Geological and Environmental Sciences, University \\ of Bologna, Viale Adriatico 1/N, 61032, Fano, Italy, \\ ${ }^{3}$ Computational Science Lab, University of Amsterdam, Science Park 904, 1098XH, Amsterdam, the Netherlands, \\ ${ }^{4}$ Marine Science Group, Department of Biological, Geological and Environmental Sciences, University of Bologna, Via Selmi 3, \\ 40126 Bologna, Italy, \\ ${ }^{5}$ Institute of Materials Physics, Helmholtz-Zentrum Geesthacht, Max-Planck-Straße 1, Geesthacht, D-21502, Germany, \\ ${ }^{6}$ Fano Marine Center, The Inter-Institute Center for Research on Marine Biodiversity, Resources and Biotechnologies, Viale \\ Adriatico 1/N 61032 Fano, Italy \\ giuseppe.falini@unibo.it
}

Otolith biomineralization results from biochemical processes regulated by the interaction of internal (physiological) and external (environmental) factors which leads to morphological and ultrastructural variability at intra- and inter-specific levels [1]. Here, for the first time, we: 1) describe the relationship between multi-scale otolith parameters and fish somatic growth (i.e., total fish length) in juveniles, females, and males of Merluccius merluccius (European hake) from the western Adriatic Sea; 2) characterize the sulcus acusticus and its subregions (ostial colliculum, caudal colliculum and collum) and measured the corresponding area and volume; 3) reveal a sexual dimorphism in the morphology of otolith during ontogenesis. We show that juvenile's otoliths had faster growth in length, width, area, perimeter, volume, weight, a higher amount of organic matter and trace element concentration, a lower density (both micro-density and bulk-density), a higher porosity and a higher value of sulcus volume: otolith volume ratio (SV:OV) compared to adult's otoliths. Furthermore, the sexual dimorphism in the morphology of otolith during ontogenesis has been revealed for the first time through a novel 3D shape analysis approach based on micro CT scans.

We found that, with increasing fish length, female saccular otoliths contained a higher amount of protuberances compared to male specimens which showed more uniform mean curvature density. The changes observed in the otolith features and sulcus acusticus regions during the growth could be linked to an eco-morphological adaptation to different biological, behavioral and environmental characteristics between juveniles and adults, which could have a functional meaning in terms of otolith response to sound waves (shape/structure-function relationships). In addition, the differences between females and males discovered in this study could be associated with fish hearing adaptation to reproductive behavioral strategies during the spawning season. Based on the outcomes of this first investigation, the use of innovative approaches is promising in highlighting differences in otoliths that could bring functional significance in specific ecological and behavioral contexts. Furthermore, the results obtained from this study can also provide inputs for further investigations aiming to understand otolith growth process according to fish size and gender and to explore the sources of otolith morphological variability during ontogenesis.

Future virtual experiments of vibroacoustic will be addressed in order to establish the shape/structure-function relationships in otoliths during fish ontogenesis and between sex and, consequently, investigate if there are any differences in the otolith response to sound waves which could enhance auditory abilities in a certain habitat or improve fish communication in specific contexts.

[1] Campana, S.E. (1992) Measurement and interpretation of the microstructure of fish otoliths. Canadian Special Publication of Fisheries and Aquatic Sciences, 117, 59-71.

Keywords: otolith; shape; morphology; physiological patterns; micro-CT 